\title{
Controlling the electromagnetic proximity effect by tuning the mixing between superconducting and ferromagnetic order
}

\author{
R. Stewart, ${ }^{1, *}$ M. G. Flokstra, ${ }^{1}$ M. Rogers, ${ }^{2}$ N. Satchell, ${ }^{2,3}$ G. Burnell,,${ }^{2}$ D. Miller, ${ }^{4}$ H. Luetkens, ${ }^{5}$ T. Prokscha, ${ }^{5}$ A. Suter, ${ }^{5}$ \\ E. Morenzoni, ${ }^{5}$ and S. L. Lee ${ }^{1}$ \\ ${ }^{1}$ School of Physics and Astronomy, SUPA, University of St Andrews, St Andrews KY16 9SS, United Kingdom \\ ${ }^{2}$ School of Physics and Astronomy, University of Leeds, Leeds LS2 9JT, United Kingdom \\ ${ }^{3}$ ISIS, Rutherford Appleton Laboratory, Oxfordshire OX11 OQX, United Kingdom \\ ${ }^{4}$ School of Chemistry, University of St Andrews, St Andrews KY16 9ST, United Kingdom \\ ${ }^{5}$ Labor für Myonspinspektroskopie, Paul Scherrer Institut, CH-5232 Villigen PSI, Switzerland
}

(Received 27 May 2019; revised manuscript received 28 June 2019; published 12 July 2019)

\begin{abstract}
We present low-energy muon-spin rotation measurements on $\mathrm{Cu} / \mathrm{Nb} / \mathrm{AlO}_{x} / \mathrm{Co}$ thin films that probe the newly described electromagnetic (EM) proximity effect. By varying the thickness of the insulating $\mathrm{AlO}_{x}$ layer we control the degree of coupling between the superconductor and ferromagnet and thus the EM proximity effect. For barrier thicknesses up to $4 \mathrm{~nm}$ we find both a small contact-dependent reduction in the standard Meissner effect and a larger diamagnetic contribution originating at the $\mathrm{Nb} / \mathrm{AlO}_{x} / \mathrm{Co}$ interface which decays away over a lengthscale far exceeding the superconducting coherence length. This second component we attribute to the EM proximity effect. Our analysis provides compelling experimental evidence for previously neglected electromagnetic effects within proximity coupled systems.
\end{abstract}

DOI: 10.1103/PhysRevB.100.020505

\section{INTRODUCTION}

The interaction between thin film conventional superconductivity (S) and ferromagnetism (F) within proximity coupled systems is one of much active interest. The transfer of electrons across $\mathrm{S} / \mathrm{F}$ interfaces gives rise to unconventional superconducting states [1-3] that can be utilized in superconducting spintronic devices $[4,5]$. This is possible due to the mixing of the opposing $\mathrm{S}$ and $\mathrm{F}$ orders having important consequences for the Cooper pairs present within the system. Ordinarily these are constructed from electrons of opposite spin and momenta but the ferromagnetic exchange field acts to align spins. The net result is a conversion of some pairs to an $s$-wave triplet state with odd frequency character. This mixing of $\mathrm{S}$ and $\mathrm{F}$ orders therefore leads to a number of interesting results such as oscillations in critical temperature $\left(T_{\mathrm{c}}\right)$ [6] and critical current [7], $\pi$ phase shifts [8,9], colossal $T_{\mathrm{c}}$ suppression [10], and long-range triplet supercurrents [11]. The presence of unconventional spin-triplet pairs has also been shown to be capable of producing a paramagnetic, as opposed to diamagnetic, Meissner response [12-14]. More recently, an anomalous flux lowering has been reported in $\mathrm{N} / \mathrm{S} / \mathrm{F}$ systems (with $\mathrm{N}$ a normal metal), over lengthscales far greater than any coherence length phenomena (e.g., inverse proximity [15-17]), which could not be understood within the existing quasiclassical theory $[18,19]$. This latter result, which at the time of its publication was unanticipated by theory, has led to the development of a new theory of electromagnetic (EM) proximity which describes how the interaction of the

*Corresponding author: rs663@st-andrews.ac.uk superconductivity with the vector potential of the $\mathrm{F}$ layer influences the screening response [20-23]. This EM proximity effect occurs at the $\mathrm{S} / \mathrm{F}$ interface, due to the penetration of the superconducting wave function into $\mathrm{F}$, and decays exponentially over a lengthscale governed by the London penetration depth $(\lambda)$ which is typically much longer than the superconducting coherence length. In order to measure directly any differences in screening behavior one is required to probe locally the magnetic flux profile across a sample. Low-energy muon-spin rotation (LE- $\mu \mathrm{SR}$ ) provides a means to achieve this and has been used extensively to probe new physics within these S/F proximity coupled systems [14,18,19,24].

In the present work the new EM proximity theory is used successfully to model the effect of the interaction between $\mathrm{S}$ and $\mathrm{F}$ on the flux profile, demonstrating that the previously anomalous effect reported in [19] can be explained within this new theoretical picture. In order to further test the theory we additionally control the degree of coupling between $S$ and $F$ through the insertion of thin insulating barriers (I) as a means to investigate how EM proximity develops as a function of the contact between $S$ and F. We utilize LE- $\mu$ SR measurements on these resultant $\mathrm{N} / \mathrm{S} / \mathrm{I} / \mathrm{F}$ thin film structures to directly probe the screening response of the proximitized system. A nonmonotonic behavior of the total flux expulsion as a function of the insulator thickness is observed that can be decomposed into two components: the effect of pair breaking on the normal Meissner screening and an EM proximity contribution. Our work highlights the need to extend theory to describe the interplay of these two proximity mechanisms and suggests the possibility to design devices where the pair breaking can be minimized while the EM proximity is maximized. 


\section{RESULTS}

For all measured samples $\mathrm{N}=\mathrm{Cu}(40 \mathrm{~nm}), \mathrm{S}=\mathrm{Nb}(50 \mathrm{~nm})$, and $\mathrm{F}=\mathrm{Co}(2.4 \mathrm{~nm})$. For the insulator we use $\mathrm{I}=\mathrm{AlO}_{x}$ of thickess $d_{\mathrm{I}}=[0,2,4,6,8] \mathrm{nm}$. The samples were grown on $\mathrm{Si}(100)$ substrates using DC magnetron sputtering at ambient temperature in a system with a base pressure of $10^{-8}$ mbar. All Co layers were grown upon a $\mathrm{Nb}(3 \mathrm{~nm})$ seed layer which in each case provided a lattice matched buffer to ensure a clean growth of the Co and therefore reduced any stray fields at the resultant $\mathrm{S} / \mathrm{I} / \mathrm{F}$ interfaces. This seed layer was too thin to become superconducting. Growth was performed under an Ar flow of 26 SCCM (SCCM denotes cubic centimeter per minute at STP) and at a typical rate of $0.2 \mathrm{~nm} \mathrm{~s}^{-1}$. All material growth rates were calibrated through fits to Kiessig fringes measured by low-angle $\mathrm{x}$-ray reflectivity. The samples were grown in two separate vacuum cycles with corresponding control samples to allow comparison between sets. The growth of all $\mathrm{AlO}_{x}$ barriers was performed, via a similar method to that used in Refs. [25,26], in stages of $2 \mathrm{~nm}$ to ensure a near uniform oxidation throughout. First, a layer of $\mathrm{Al}(2 \mathrm{~nm})$ was deposited before being exposed to an $\mathrm{O}_{2}$ flow of $76 \mathrm{SCCM}$ for $60 \mathrm{~s}$. This process was repeated $n$ times until the desired thickness of oxide had been deposited. Following oxide deposition the chamber vacuum pressure was restored before any further growth occurred. Figure 1 shows a transmission electron microscopy cross section of the sample with $d_{\mathrm{I}}=2 \mathrm{~nm}$. The bright and dark areas correspond to metallic and insulating layers, respectively. It can be seen that the $\mathrm{AlO}_{x}$ layer is uniformly deposited and oxidized throughout.

All LE- $\mu$ SR measurements were carried out on the $\mu \mathrm{E} 4$ beamline at the Paul Scherrer Institut [27]. Low -energy muons provide the means to measure the magnetic flux profile across a thin film with a possible field uncertainty of less than $0.1 \mathrm{G}$ [28]. A stream of nearly $100 \%$ spin-polarized muons, unstable spin- $\frac{1}{2}$ leptons of charge $+e$ and lifetime $2.197 \mu \mathrm{s}$, is incident on the sample. In order to probe the screening behavior an external magnetic field is applied within the plane of the sample but orthogonal to the plane containing the muon spin. This is the transverse field geometry. Upon implantation a muon will rapidly thermalize while preserving its initial spin direction. It will then begin to precess around the local magnetic field $\left(B_{\text {loc }}\right)$ at a frequency $\omega_{\mu}=\gamma_{\mu} B_{\text {loc }}$ where $\gamma_{\mu}=$

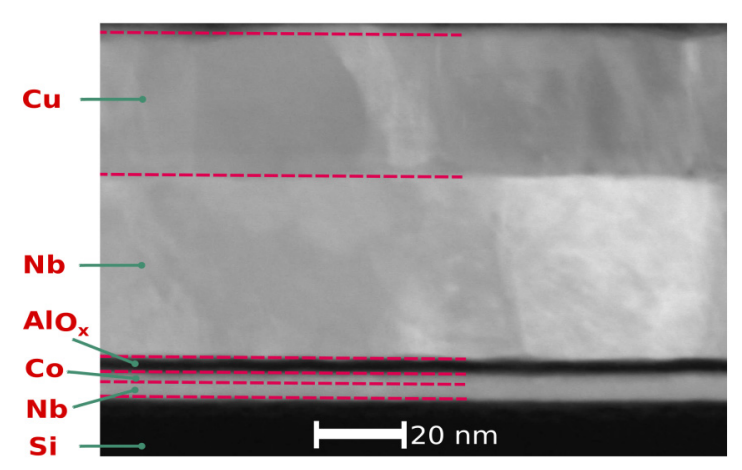

FIG. 1. Sample transmission electron microscopy image showing the $\mathrm{Cu} / \mathrm{Nb} / \mathrm{AlO}_{x}(2 \mathrm{~nm}) / \mathrm{Co}$ sample. The insulating layers are shown in black and the conducting layers are bright.
$2 \pi * 135.5 \mathrm{MHz} \mathrm{T}^{-1}$ is the muon gyromagnetic ratio. Some time after implantation the muon will decay into a positron that is emitted preferentially along the momentary muon-spin direction. By monitoring the emitted decay positrons for a statistically significant number of events information about the precession frequency and therefore the local field experienced can be obtained. The depth at which a muon comes to rest within the sample is energy dependent. By altering the energy of the incoming muons one can tune the average probing depth between about 10 and $100 \mathrm{~nm}$ below the sample surface. A typical experiment involves measuring at a series of different muon energies, where for each energy several million positron counts are collected at a rate of around $1000 \mathrm{~s}^{-1}$, such that for each energy, $E$, the average flux, $\langle B\rangle(E)$, can be determined. Given an incident muon energy, a well-proven Monte Carlo algorithm can be used to determine the implantation depth profile (stopping profile) for a given sample material $[29,30]$. This allows a conversion from $\langle B\rangle(E)$ to $\langle B\rangle\langle x\rangle$ where $\langle x\rangle$ is the average probing depth for muons with implantation energy $E$. This is the standard approach to the data analysis and gives a first indication of the spatial profile of flux across the sample. In cases where the underlying field profile is known, however, the analytical form of $B(x)$ can be imposed on the measured data to obtain a best fit $B(x)$ for all measurement energies simultaneously.

For a single $\mathrm{Nb}$ thin film with vacuum interfaces the expected flux profile follows directly from the London equation and is given by

$$
B_{\mathrm{S}}(x)=B_{0} \cosh \left(\frac{x}{\lambda}-\frac{d_{\mathrm{S}}}{2 \lambda}\right) \cosh \left(\frac{d_{\mathrm{S}}}{2 \lambda}\right)^{-1}
$$

where $d_{\mathrm{S}}$ is the $\mathrm{Nb}$ thickness and $x=0$ corresponds to the top surface of the film [31]. While in principle an extension to the $\mathrm{N} / \mathrm{S}$ bilayer case could be obtained by using the quasiclassical theory to calculate a numerical profile for the screening response, we find for our combination of sample material parameters the $\mathrm{Cu}$ becomes fully proximitized and screening develops across the full spatial extent of the bilayer in a nearly symmetric manner [19]. The resultant profile therefore becomes $B_{\mathrm{NS}}(x)=B_{\mathrm{S}}(x)$ but where we now use the full thickness of the bilayer, $d_{\mathrm{NS}}$, in the model. The addition of a ferromagnetic layer in direct contact with the $\mathrm{Nb}$ will have several measurable consequences for the shape of the flux profile. Firstly, it will suppress the superconducting condensate within the region close to the $\mathrm{S} / \mathrm{F}$ interface. This will result in a decrease of the standard Meissner response to one that we now label $B_{\mathrm{NS}}^{*}$. We account for this reduction in our model through the use of an effective penetration depth, $\lambda_{\text {eff }}$, which will be longer than the $\lambda$ of the bilayer. Secondly, in the case of the N/S/F and N/S/I/F samples we include the effect of EM proximity which involves the spontaneous generation of screening currents due to the magnetic vector potential at (or near) the $S / F$ interface resulting in a magnetic flux within the superconductor. To account for the EM proximity effect we add an additional term to the model flux profile which represents an amplitude originating at the $\mathrm{S} / \mathrm{I} / \mathrm{F}$ interface that decays away over $\lambda_{\text {eff }}$ as in [21]

$$
B_{\mathrm{NSIF}}(x)=B_{\mathrm{NS}}^{*}+A_{\mathrm{EM}} e^{\left(x-d_{\mathrm{NS}}\right) / \lambda_{\mathrm{eff}}},
$$




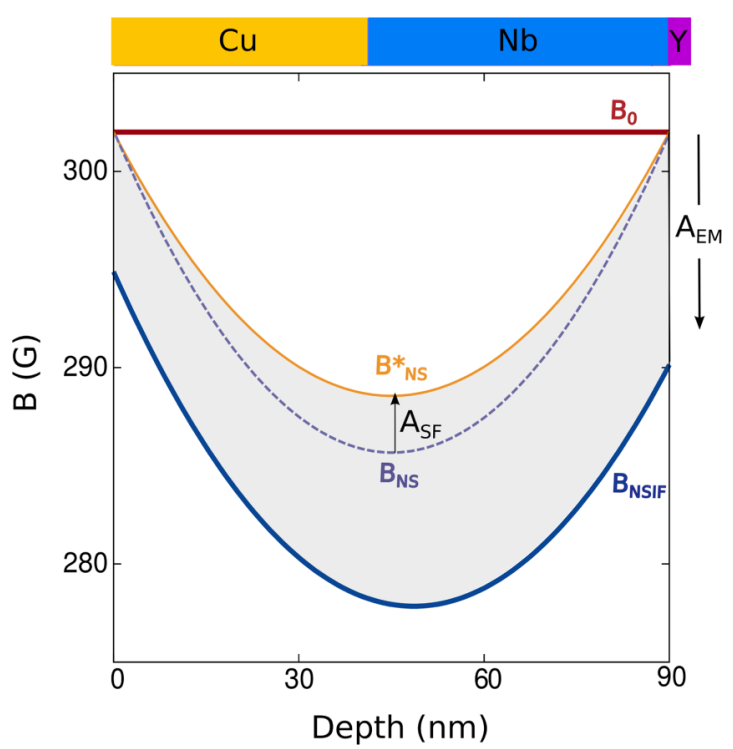

FIG. 2. A schematic of the different field profiles. $B_{0}$ is the constant normal state flux density and $B_{\mathrm{NSIF}}$ the superconducting state profile given by Eq. (2). $B_{\mathrm{NSIF}}$ can be decomposed into an EM contribution (shaded region) and a standard Meissner contribution $\left(B_{\mathrm{NS}}^{*}\right)$ determined from the bilayer response $\left(B_{\mathrm{NS}}\right)$ by including pair breaking. $A_{\mathrm{EM}}$ and $A_{\mathrm{SF}}$ are the EM and pair-breaking amplitudes, respectively.

where $A_{\mathrm{EM}}$ is the amplitude of the electromagnetic proximity effect. In summary the form of Eq. (2), which describes the flux profile for the $\mathrm{N} / \mathrm{S} / \mathrm{F}$ and $\mathrm{N} / \mathrm{S} / \mathrm{I} / \mathrm{F}$ samples, therefore comprises two terms. The first describes the standard Meissner screening of the externally applied magnetic field and the additional second term accounts for the electromagnetic proximity effect as recently proposed in Ref. [21].

Figure 2 shows a schematic of the field profile given by Eq. (2). $B_{0}$ indicates the constant normal state flux density due to the applied measurement field and $B_{\mathrm{NSIF}}$ shows the full superconducting state profile which is broken down into its constituent components: the normal Meissner part $\left(B_{\mathrm{NS}}^{*}\right)$ and the EM part (shaded region). $A_{\mathrm{SF}}$ parametrizes the effect of pair breaking found from the difference between $B_{\mathrm{NS}}$ and $B_{\mathrm{NS}}^{*}$. In order to model the data we take Eq. (2) and allow both the amplitude of the EM contribution and the value of $\lambda_{\text {eff }}$ to vary as free fit parameters such that the imposed profile best predicts the measured flux averages.

Figure 3 presents the results of this approach to analyzing the LE- $\mu$ SR data for a set of samples of the form $\mathrm{Cu}(40) / \mathrm{Nb}(50) / Y$ where $Y=\mathrm{Si}, \mathrm{Co} / \mathrm{Si}$, and $\mathrm{AlO}_{x}(2 \mathrm{~nm}) / \mathrm{Co} / \mathrm{Si}$ for the N/S, N/S/F, and N/S/I/F samples, respectively. The top panel shows the muon stopping profiles for a range of different energies. At $4 \mathrm{keV}$, the lowest sampled muon energy, all muons stop exclusively within the $\mathrm{Cu}$ layer. As the muon energy increases the stopping distribution shifts to higher average depths while a tail of the distribution persists within the $\mathrm{Cu}$. The solid lines in the bottom panel show the result of imposing the underlying field profiles, given by Eq. (1) for the N/S sample and Eq. (2) for the $\mathrm{N} / \mathrm{S} / \mathrm{F}$ and $\mathrm{N} / \mathrm{S} / \mathrm{I} / \mathrm{F}$ samples, on the measured data. The circles in the bottom panel represent the results of the

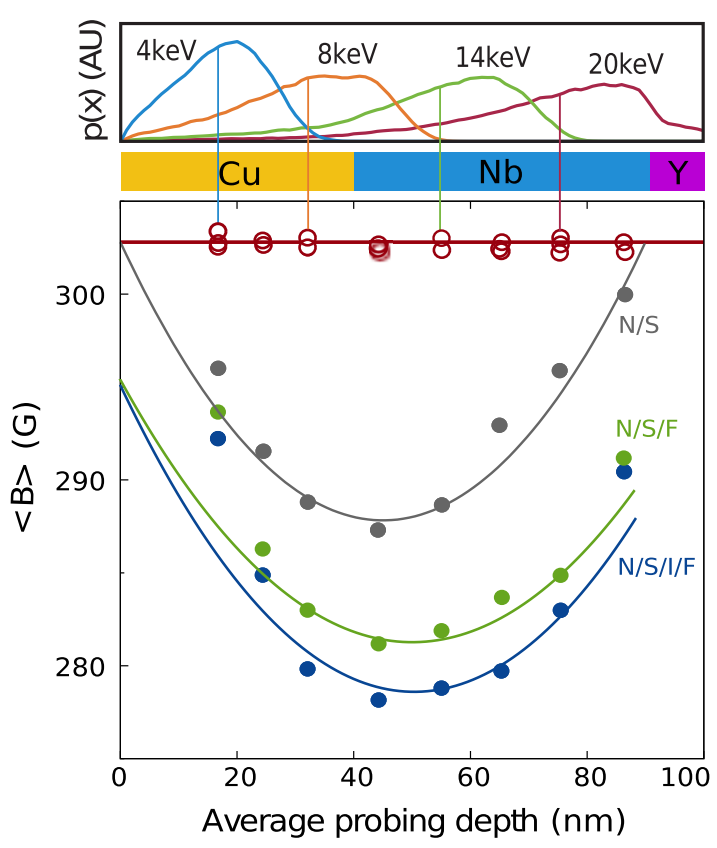

FIG. 3. Top panel: Muon stopping profiles for several selected implantation energies. The profiles for $E \geqslant 20 \mathrm{keV}$ extend into the $Y$ layers. A schematic of the sample structure is shown for reference. Bottom panel: Example results of the LE- $\mu$ SR measurements for an applied field of around $300 \mathrm{G}$. The average field values as a function of average depth are plotted as points, above and below $T_{\mathrm{c}}$, for a range of samples of the form $\mathrm{Cu}(40) / \mathrm{Nb}(50) / Y$ where $Y=$ $\mathrm{Si}, \mathrm{Co} / \mathrm{Si}, \mathrm{AlO}_{x}(2 \mathrm{~nm}) / \mathrm{Co} / \mathrm{Si}$ for the N/S, N/S/F, and N/S/I/F samples, respectively. All normal (superconducting) state data were measured at $10 \mathrm{~K}(2.5 \mathrm{~K})$ and are plotted in open (closed) symbols. The error bars are $0.1-0.3 \mathrm{G}$ and are thus smaller than the symbol size. The solid lines represent the best-fit imposed field profiles which determine the full spatial dependence.

$\langle x\rangle$ approach to the data analysis. The data plotted with open symbols correspond to the $T=10 \mathrm{~K}$ normal state measurements and the closed symbols the $T=2.5 \mathrm{~K}$ superconducting state measurements. Error bars in the average field values are plotted in each case and typically fall within $0.1-0.3 \mathrm{G}$, which is smaller than the symbol size. In the case of the normal state data for all samples we simply recover the applied field, which was set to around 300 Oe (the maximum possible at the beamline in this measurement geometry). In the superconducting state, measured at $2.5 \mathrm{~K}$, in all samples we observe a lowering of the measured flux within the sample. The N/S data show Meissner screening behavior extending into the copper. As previously reported in [19] the addition of a thin ferromagnetic layer $(\mathrm{N} / \mathrm{S} / \mathrm{F})$ in direct contact with the superconductor results in a significant increase to the observed flux expulsion. The N/S/I/F data shows an overall enhancement to the normal Meissner part of the signal when compared to the N/S/F case while the EM part appears to remain largely constant. This suggests the degree of coupling has been altered sufficiently to affect the pair breaking, and consequently the standard mesoscopic Meissner response, but not the EM contribution. For all measured samples we find a good agreement between the two different approaches to the data analysis. The conventional averages approach captures 


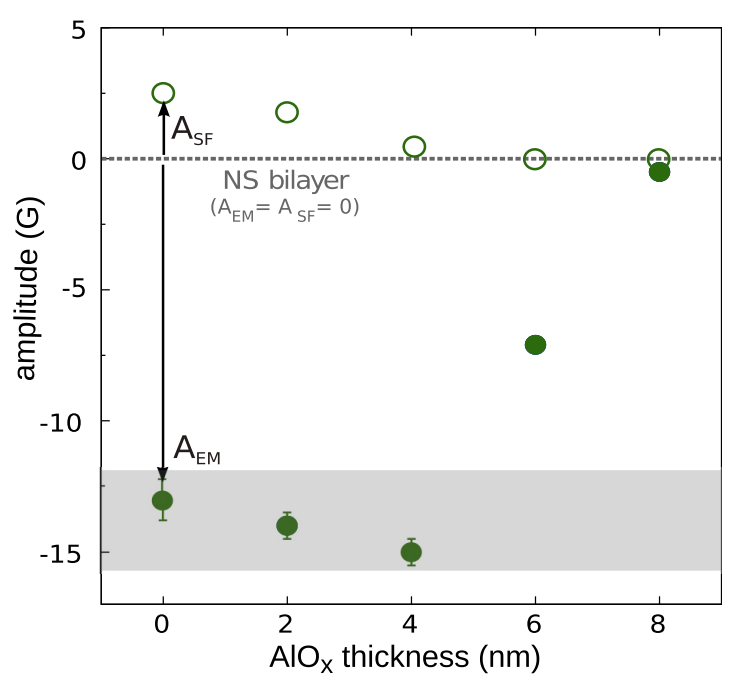

FIG. 4. The extracted proximity amplitudes as a function of $d_{\mathrm{I}}$. The open and closed data points represent the extracted $A_{\mathrm{SF}}$ and $A_{\text {EM }}$ amplitudes, respectively. The horizontal dotted line represents the NS bilayer for which both amplitude contributions are zero. The light shaded region indicates a relatively constant "band" of $A_{\mathrm{EM}}$ amplitudes for $d_{\mathrm{I}} \leqslant 4 \mathrm{~nm}$.

the same trends as the full spatial analysis but by imposing the field profiles we are able to identify and extract the physical parameters of the system and see how these change with the strength of coupling between $\mathrm{S}$ and $\mathrm{F}$.

In order to track both the effects of pair breaking and EM proximity as a function of the coupling between $\mathrm{S}$ and $\mathrm{F}$, we measure the full range of $\mathrm{AlO}_{x}$ thicknesses and model the spatial profile for each sample. In each case we compare the resulting proximity amplitudes with those of the bilayer control sample. Figure 4 shows the extracted proximity amplitudes as a function of the oxide layer thickness. The error bars are calculated to account for both the data fitting and any sampleto-sample variations in $T_{\mathrm{c}}-T_{\mathrm{S}}$, where $T_{\mathrm{S}}$ is the temperature at which the measurement was conducted (around $2.5 \mathrm{~K}$ ). The extracted $A_{\mathrm{SF}}$ values (open symbols) show that when the $\mathrm{Nb}$ and $\mathrm{Co}$ are in direct contact with one another there is a small reduction of approximately 15\% in the standard Meissner response. As the degree of coupling is reduced, through the insertion of insulating barriers, this suppression in screening diminishes such that for $d_{\mathrm{I}}>4 \mathrm{~nm}$ the NS bilayer result is recovered. On the other hand, $A_{\mathrm{EM}}$ (closed symbols) appears to take an approximately constant value of $(-14 \pm 1) \mathrm{G}$ for $d_{\mathrm{I}}<4 \mathrm{~nm}$. Further increase in the barrier thickness causes the EM amplitude to die off.

\section{DISCUSSION AND CONCLUSIONS}

The modeling and spatial dependence of the LE- $\mu$ SR data is consistent with there being an additional source of vector potential at the $\mathrm{S} / \mathrm{I} / \mathrm{F}$ interface that unambiguously requires the presence of a ferromagnet. The resulting orbital response appears to be related to the direct sampling of $\mathrm{F}$ by the superconducting condensate rather than stray fields since for thick insulating layers the bilayer result is recovered. Theory suggests the local magnetization of the $\mathrm{F}$ layer is the source of the vector potential. This is highly consistent with our measurements that clearly show the additional screening originates only from one side of the sample, namely, the ferromagnetic layer, and disappears once that layer is fully isolated. The origin of the approximately constant value of $A_{\mathrm{EM}}$ for $d_{\mathrm{I}} \leqslant 4 \mathrm{~nm}$ is currently unknown and is not easily discerned from the existing theory. Intuitively $A_{\mathrm{EM}}$ would be related both to the sampling of $\mathrm{F}$ by $\mathrm{S}$ (which we gradually decouple by introducing the insulating layer) and to the gap strength at the interface (which becomes larger for thicker barrier layers due to the reduced pair-breaking effect). Since the scenario with an insulating barrier is not currently explored explicitly by the presently published theory, the precise nature of this dependence remains an open question. Our results, however, suggest the possibility of a device in which the direct proximity, $A_{\mathrm{SF}}$, is minimal while the EM effect, $A_{\mathrm{EM}}$, remains maximal. We note also that while the asymmetric screening strongly supports the influence of a localized source of vector potential, there may be other contributions, for example, Rashba-type spin-orbit effects connected with the interface, which may also play a role. Further theoretical developments are likely to suggest new measurements to test stringently the details of alternative scenarios. Muon experiments in zero applied field may become important to help distinguish between various contributions.

In conclusion, we have studied the influence of isolating oxide layers on the previously reported anomalous enhancement of diamagnetic screening due to the presence of a thin ferromagnetic layer. We analyze the data using a model consistent with the theory of the recently described EM proximity effect and find two opposing contributions that act to alter the standard Meissner profile, one originating from the anticipated pair-breaking influence and the other the EM proximity effect, both of which are ultimately suppressed by a thick insulating oxide barrier. We find, for our combination of sample parameters, the EM component to be both persistently diamagnetic and substantially larger than the opposing effect of pair breaking. In general, we find good agreement between theory and experiment providing strong evidence for newly described proximity effects within S/F systems. Understanding and exploiting these effects is likely to be extremely important in the field of superconducting spintronics, and the present work provides a framework in which to interpret a number of earlier relevant and seemingly anomalous results in the field $[18,19]$.

\section{ACKNOWLEDGMENTS}

The authors wish to thank A. Buzdin and B. Braunecker for useful discussions. The work was supported financially through the following EPSRC grants: Grants No. EP/I031014/1, No. EP/J01060X, No. EP/J010634/1, No. EP/R031924/1, No. EP/R023522/1, No. EP/L015110/1, and No. EP/L017008/1 and has received funding through the European Union's Horizon 2020 Research and Innovation Programme under the Marie Skłodowska-Curie Grant Agreement No. 743791 (SUPERSPIN). All muon measurements presented in this work were carried out on the LEM instrument courtesy of the Paul Scherrer Institut. 
[1] T. Löfwander, T. Champel, J. Durst, and M. Eschrig, Phys. Rev. Lett. 95, 187003 (2005).

[2] A. I. Buzdin, Rev. Mod. Phys. 77, 935 (2005).

[3] F. S. Bergeret, A. F. Volkov, and K. B. Efetov, Rev. Mod. Phys. 77, 1321 (2005).

[4] M. Eschrig, Rep. Prog. Phys. 78, 104501 (2015).

[5] J. Linder and J. W. A. Robinson, Nat. Phys. 11, 307 (2015).

[6] J. S. Jiang, D. Davidović, D. H. Reich, and C. L. Chien, Phys. Rev. Lett. 74, 314 (1995).

[7] Y. Blum, A. Tsukernik, M. Karpovski, and A. Palevski, Phys. Rev. Lett. 89, 187004 (2002).

[8] T. Kontos, M. Aprili, J. Lesueur, and X. Grison, Phys. Rev. Lett. 86, 304 (2001).

[9] V. V. Ryazanov, V. A. Oboznov, A. Yu. Rusanov, A. V. Veretennikov, A. A. Golubov, and J. Aarts, Phys. Rev. Lett. 86, 2427 (2001).

[10] A. Singh, S. Voltan, K. Lahabi, and J. Aarts, Phys. Rev. X 5, 021019 (2015).

[11] R. S. Keizer, S. T. B. Goennenwein, T. M. Klapwijk, G. Miao, G. Xiao, and A. Gupta, Nature (London) 439, 825 (2006).

[12] T. Yokoyama, Y. Tanaka, and N. Nagaosa, Phys. Rev. Lett. 106, 246601 (2011).

[13] M. Alidoust, K. Halterman, and J. Linder, Phys. Rev. B 89, 054508 (2014).

[14] A. Di Bernardo, Z. Salman, X. L. Wang, M. Amado, M. Egilmez, M. G. Flokstra, A. Suter, S. L. Lee, J. H. Zhao, T. Prokscha, E. Morenzoni, M. G. Blamire, J. Linder, and J. W. A. Robinson, Phys. Rev. X 5, 041021 (2015).

[15] V. N. Krivoruchko and E. A. Koshina, Phys. Rev. B 66, 014521 (2002).

[16] F. S. Bergeret, A. F. Volkov, and K. B. Efetov, Phys. Rev. B 69, 174504 (2004).

[17] F. S. Bergeret, A. Levy Yeyati, and A. Martín-Rodero, Phys. Rev. B 72, 064524 (2005).
[18] M. G. Flokstra, N. Satchell, J. Kim, G. Burnell, P. J. Curran, S. J. Bending, J. F. K. Cooper, C. J. Kinane, S. Langridge, A. Isidori, N. Pugash, M. Eschrig, H. Luetkens, A. Suter, T. Prokscha, and S. L. Lee, Nat. Phys. 12, 57 (2016).

[19] M. G. Flokstra, R. Stewart, N. Satchell, G. Burnell, H. Luetkens, T. Prokscha, A. Suter, E. Morenzoni, S. Langridge, and S. L. Lee, Phys. Rev. Lett. 120, 247001 (2018).

[20] F. S. Bergeret, A. F. Volkov, and K. B. Efetov, Europhys. Lett. 66, 111 (2004).

[21] S. Mironov, A. S. Mel'nikov, and A. Buzdin, Appl. Phys. Lett. 113, 022601 (2018).

[22] A. F. Volkov, F. S. Bergeret, and K. B. Efetov, Phys. Rev. B 99, 144506 (2019).

[23] Z. Devizorova, S. V. Mironov, A. S. Mel'nikov, and A. Buzdin, Phys. Rev. B 99, 104519 (2019).

[24] M. G. Flokstra, S. J. Ray, S. J. Lister, J. Aarts, H. Luetkens, T. Prokscha, A. Suter, E. Morenzoni, and S. L. Lee, Phys. Rev. B 89, 054510 (2014).

[25] H. X. Wei, M. C. Hickey, G. I. R. Anderson, X. F. Han, and C. H. Marrows, Phys. Rev. B 77, 132401 (2008).

[26] M. S. Gabureac, K. J. Dempsey, N. A. Porter, C. H. Marrows, S. Rajauria, and H. Courtois, J. Appl. Phys. 103, 07A915 (2008)

[27] T. Prokscha, E. Morenzoni, K. Deiters, F. Foroughi, D. George, R. Kobler, A. Suter, and V. Vrankovic, Nucl. Instrum. Methods Phys. Res., Sect. A 595, 317 (2008).

[28] P. Bakule and E. Morenzoni, Contemp. Phys. 45, 203 (2004).

[29] W. Eckstein, Computer Simulation of Ion-Solid Interactions (Springer, Berlin/Heidelberg, 1991).

[30] E. Morenzoni, H. Glückler, T. Prokscha, R. Khasanov, H. Luetkens, M. Birke, E. M. Forgan, C. Niedermayer, and M. Pleines, Nucl. Instrum. Methods, Phys. Res., Sect. B 192, 254 (2002).

[31] A. C. Rose-Innes and E. H. Rhoderick, Introduction to Superconductivity (Pergamon, New York, 1994). 